\title{
EL PROCESO DE CONSTRUCCIÓN DEL NUEVO DISEÑO CURRICULAR PARA EL PROFESORADO DE EDUCACIÓN PRIMARIA Nº 528/09 EN LA PROVINCIA DE SANTA FE
}

\author{
Mauro Carbonari \\ Camila Carlachiani \\ Romina Craparo*
}

\section{Resumen}

La principal temática en torno a la cuál se vertebra el presente artículo es la formación docente, tomando como objeto de estudio al proceso de Cambio Curricular que se llevó a cabo en la carrera de Profesorado para Educación Primaria de la Provincia de Santa Fe durante el año 2008.

Dentro de este marco, se encuentra el proceso de Cambio Curricular mencionado. La presente investigación, en clave de aportar herramientas para pensar las relaciones entre los distintos espacios de poder y de formación que se dan dentro del campo del currículum, tiende a focalizar la mirada en la participación y las tensiones que se desataron entre los distintos actores curriculares a lo largo de dicho proceso.

\section{Palabras Clave}

Formación docente - Cambio curricular - Currículum - Participación simbólica - Tensiones disciplinas clásicas/espacios innovadores.

\begin{abstract}
The main theme around which this article is the backbone of teacher education, taking as a case study the process of curriculum change that took place in the race for Primary Teachers in the Province of Santa Fe during the 2008. Within this framework is the process of curriculum change mentioned. This research, key to providing tools for thinking the relationship between different spaces of power and given training in the field of curriculum tends to focus his eyes on the participation
\end{abstract}

* Estudiantes de la carrera de Prof. y Lic. en Ciencias de la Educación, Facultad Humanidades y Artes. UNR. Trabajo avalado por la Dra. Marta Brovelli, Profesora Titular del Área Curriculum I y II. Ciencias de la Educación UNR. 
and the tensions that erupted between the various actors curricular throughout this process.

\section{Key Words}

Teacher education - Curricular change - Curriculum - Participation symbolic - Tension classical disciplines / innovative spaces.

\section{Introducción}

El presente artículo es parte de un proyecto de investigación que se enmarca como trabajo final del Área del Currículum durante el año 2009, de la carrera de Ciencias de la Educación de la Facultad de Humanidades y Artes de la Universidad Nacional de Rosario.

El principal objetivo de este artículo es aportar al conocimiento de un proceso de construcción curricular al cuál concebimos como un campo de lucha (De Alba, 2008). Dicho aporte pretende ser una herramienta de apertura para la comprensión de los cambios que suceden en la formación docente en la actualidad.

El cambio curricular analizado surge como iniciativa del Instituto Nacional de Formación Docente (INFOD), el cual sostiene que las prácticas profesionales son prácticas sociales bañadas de incertidumbres, pero que deben permitir construir contornos sociales (De Alba, 2008) que sustenten el derecho a enseñar y a aprender. Para el INFOD las instituciones son concebidas de origen social y político, por lo tanto son susceptibles de cambio, planteando procesos de planificación y reflexión continua. Por ello, asume la función de planificar y ejecutar políticas de articulación del sistema formador docente inicial y continua conforme a lo establecido por la Ley de Educación Nacional N²6.206/06.

El presente artículo cuenta con cuatro ejes fundamentales que constituyen, a la vez que ordenan, el análisis de todo el material recogido a través del trabajo de campo realizado.

\section{Relaciones entre los Lineamientos Curriculares Nacionales para Formación Docente y el Diseño Curricular Jurisdiccional}

El proceso de cambio curricular llevado a cabo en la Provincia de Santa Fe durante el año 2008 tuvo como referencia algunas cuestiones básicas prescriptas desde el INFOD a través de los Lineamientos Curriculares Nacionales para la Formación Docente Inicial y Continua (LCN). Una de las disposiciones de este organismo, prescribe que todas las carreras de formación docente deben tener una extensión de cuatro años de duración para ser acreditadas por la administración nacional. Esta es una de las razones por la cuál comenzó a plantearse la iniciativa de realizar una reforma en el Diseño Curricular para el Profesorado de Educación Primaria de la Provincia de Santa Fe. 
En el discurso de los profesores de los Institutos de Formación Docente (IFD) este cambio curricular surge como una necesidad de los mismos. Además, gran parte de los estudiantes encuestados de los IFD, postularon que la extensión a cuatro años de la carrera docente se justifica porque al agregar un año más a la misma, la residencia se haría menos agobiante ya que no habría que cursar simultáneamente otras unidades curriculares.

Podríamos inferir entonces que, desde el marco normativo, la propuesta de cambio curricular surge de la Administración Nacional pero, si pensamos al currículum como un proyecto ético-político socialmente construido, afirmaríamos que esta norma no hubiese sido posible sin la existencia de la demanda de los IFD y de sus actores institucionales.

Los campos de formación necesarios en lo que respecta a la formación docente también son prescriptos por los LCN: Campo de la Formación General, Campo de la Formación Específica y Campo de la Formación en la Práctica Profesional. Estos campos se encuentran como ejes estructurantes dentro del nuevo diseño curricular respetando los porcentajes que, según dichos LCN, debe ocupar cada uno de ellos en la formación.

\section{Proceso de construcción del nuevo diseño}

Uno de los ejes fundantes al interior del proceso de construcción del nuevo diseño curricular para el Profesorado de Educación Primaria No 528/09 de la Provincia de Santa Fe es el de la participación. En este sentido, podríamos vislumbrar que existen diversos discursos y posturas acerca de dicha cuestión.

La Ministra de Educación de la Provincia postula, en una carta adjunta al diseño, que el proceso de construcción del mismo “... es el resultado de un esfuerzo colectivo verdaderamente intenso y fructífero en el cual han participado especialistas invitados, docentes del nivel a los que se relevó para la producción de los documentos de base y los propios IFD, a través de sus equipos y estudiantes". A partir de esta afirmación analizaremos el grado de participación que tuvieron los diferentes actores curriculares durante el proceso de cambio.

Con respecto a la participación de los docentes de los IFD, los integrantes de la Comisión de Cambio Curricular manifiestan que se trató de un proceso participativo. Sin embargo, este proceso participativo luego se iría transformando y dejando a un lado. En palabras de una regente de nivel primario: "Sí, participamos en el análisis crítico de los planes. En la primera parte donde necesitaban que contáramos la experiencia, pero después ya vino hecho el plan..."Así, se reflejaría un proceso de participación simbólica (Sirvent, 1985:45) (1) puesto que desde la Comisión de Cambio Curricular se les otorgó a los IFD un poder de decisión que luego se tornó inexistente.

Con respecto a la participación que tuvieron los estudiantes de los IFD se pueden observar dos posturas totalmente opuestas. Por un lado, en uno de los IFD, pareciera que los estudiantes sí tuvieron participación en el proceso de 
cambio curricular. En cambio, en otro establecimiento, una docente sostiene que no tuvieron ningún tipo de participación. Reafirmando esto último, en las encuestas realizadas sólo un estudiante manifiesta haber tenido participación, el cuál aclara que la misma consistía en un taller informativo que no implicaba una instancia reflexiva y propositiva.

Ahora bien, la responsabilidad de generar una participación real incluyendo a todos los actores institucionales en el proceso de cambio curricular no debería recaer sólo sobre la comisión a cargo del mismo, sino que los IFD deberían también asumir parte de dicha responsabilidad. Los mismos, a través de sus miembros, tienen la capacidad de ejercer poder en todos los aspectos de la vida institucional y en la toma de decisiones curriculares en los diferentes niveles de concreción.

En el proceso de cambio curricular investigado, los IFD tuvieron autonomía para organizar la forma de implementar un determinado tipo de participación. En uno de los IFD se formaron comisiones específicas para tratar el diagnóstico de los planes anteriores. En cambio, en otro el tema del cambio curricular sólo se trataba en reuniones plenarias. Las diversas formas de organizar la participación marcarían una diferencia, no sólo en la manera que posee cada IFD de concebir a la misma, sino también en la relevancia que tiene para la institución el hecho de participar e incidir en su propio proceso de cambio curricular.

Teniendo en cuenta otro plano de análisis, participaron dentro de la Comisión de Cambio Curricular especialistas de diferentes disciplinas y áreas. Esto habría generado una de las tensiones más relevantes en lo que fue el proceso de construcción del nuevo DCJ.

La concepción de curriculum que subyacía en las discusiones entre los cientistas de la educación y los especialistas de las áreas de educación artística y educación física marcaba diferencias al momento de discutir y diseñar la estructura y la organización curricular. En este sentido, una de las especialistas que conformaba dicha comisión, refiriéndose a los cientistas de la educación, manifiesta que "... mientras ellos ven al currículum como un campo de lucha donde hay ganadores y perdedores, nosotros creemos que el curriculum es un lugar de encuentro, donde se dialoga y se llega a un consenso..." En contraposición a ésto, los cientistas de la educación sostienen que el curriculum es un campo de lucha (De Alba, 2008) donde, a menudo, cada especialista privilegiaría su espacio curricular disciplinar en detrimento de la Formación General.

Por otra parte, los especialistas atribuyen la causa de la división generalistas-especialistas, dentro de la Comisión de Cambio Curricular, a que los cientistas de la educación priorizan la formación en las disciplinas acusándolos, así, de racionalistas. Además, esta concepción estaría avalada por el Ministerio de Educación de la Provincia de Santa Fe, el cuál tuvo gran participación e incidencia en las decisiones que tomaba dicha comisión. Este sostén por parte del Ministerio desnivelaba las posiciones a favor de los especialistas. 
Finalmente, luego de los encuentros y desencuentros, discusiones y conflictos desarrollados a lo largo del analizado proceso de construcción curricular nos encontramos con dos versiones opuestas acerca del desenlace del mismo.

Por un lado, se manifiesta que el diseño presentado en noviembre del 2008 por la Comisión de Cambio Curricular no fue aprobado y parte de dicha comisión cesó en sus funciones en relación al Ministerio de Educación de la Provincia, desde el cuál se modificó el diseño y se agregaron espacios curriculares al mismo. Por otro lado, existe una parte de la comisión que sostiene que la misma, luego de la presentación de los borradores del nuevo diseño, siguió trabajando en la revisión del mismo a través de reelaboraciones, aportes y nuevas escrituras. Esta última versión fue la que se dio a conocer oficialmente a través de los medios de comunicación.

A pesar de que estas dos versiones se desdicen en varios puntos, las mismas coincidirían en que se respetó gran parte de la escritura de la primera versión acabada del nuevo diseño, aunque por motivos diferentes. Uno de ellos apuntaría a no generar, desde el Ministerio de Educación de la Provincia, nuevos conflictos con los IFD. En este sentido, la coordinadora de la Comisión de Cambio Curricular nos relata que "... la Ministra dijo: "bueno, es enciclopedista pero es lo que quisieron las instituciones". Otro de los motivos aludiría a que quiénes participaron en las últimas modificaciones, en su mayoría, eran especialistas en las áreas de educación física y educación artística, ausentándose quienes pertenecían al campo de las Ciencias de la Educación.

\section{Diseño de Profesorado de Educación Primaria N 528/09: su estructura profunda y el correlato en la estructura superficial. Algunas características relevantes.}

En la estructura profunda del presente diseño se toma un posicionamiento epistemológico desde el Paradigma de la Complejidad. No sólo porque en el mismo se explicita, sino también porque desde el Ministerio de Educación de la Provincia se organizaron jornadas y talleres destinados a los docentes de los IFD, abordando teóricamente los conceptos claves de la estructura profunda desde dicha postura epistemológica.

Esta elección se justificaría debido a que los demás paradigmas se encuentran desfasados y por esta razón, el Paradigma de la Complejidad sería el indicado para abordar la realidad actual en la que los egresados de los IFD van a ejercer su profesión.

No obstante, según los datos recogidos de las entrevistas realizadas, parecería que la fundamentación del DCJ no fue clara para todos los integrantes de la Comisión de Cambio Curricular ni para los docentes de los IFD, lo cuál podría atribuirse a que realmente no se realizó un proceso de construcción colectivo. 
A partir de esto podríamos inferir que el marco teórico del diseño curricular generó cierta incertidumbre en algunos de los miembros de la mencionada comisión por lo cuál no pudieron comprenderlo en su totalidad. Frente a este hecho, quiénes quedaron conformes con la elaboración del marco teórico por parte del Ministerio de Educación de la Provincia, afirman que aquéllos no lo entendieron por su propia formación profesional disciplinaria y tradicional.

Entonces, si repasamos el fundamento epistemológico que el diseño curricular analizado posee y las categorías resaltadas en el mismo, podríamos inferir que éste se condice con algunos espacios curriculares innovadores que el mismo presenta. Por esta razón, el marco teórico guardaría mayor coherencia con dichos espacios que con las demás unidades curriculares del diseño.

De este modo, con la intención de comprobar el correlato entre la estructura profunda y la estructura superficial del nuevo Diseño Curricular para el Profesorado de Educación Primaria N 528/09 de la Provincia de Santa Fe, pasaremos a detallar los espacios innovadores que constan en la estructura superficial del mismo: Movimiento y Cuerpo, Itinerarios por el Mundo de la Cultura y Taller de Producción Pedagógica.

En primer lugar, la unidad curricular Movimiento y Cuerpo sería una de las más controvertidas ya que refleja las concepciones de conocimiento, enseñanza y aprendizaje que posee el Ministerio de Educación de la Provincia conjuntamente con los especialistas que integraron la Comisión de Cambio Curricular. Estas concepciones parten de enseñar-aprender experiencialmente desde el cuerpo a través del movimiento, para luego abordar, desde dicha perspectiva, el conocimiento disciplinar. Frente a esto, los docentes de los IFD sostienen que, si bien dicha unidad constituye una innovación para la formación docente, sería necesario, en primer lugar, formar a los estudiantes en conocimientos disciplinares

Según los mismos docentes, la discusión sobre los conocimientos disciplinares se dejaría de lado no por una cuestión intencional sino por un desconocimiento de las características cotidianas de los IFD por parte de los especialistas que conformaron la Comisión de Cambio Curricular. Esta extrañes proporcionaría un análisis erróneo acerca de la realidad de los IFD.

Ahora bien, podríamos delinear dos hipótesis posibles a partir del hecho de que Movimiento y Cuerpo no posee contenidos específicos dentro del diseño. Por un lado, dicha unidad curricular sería un cimiento, una base para luego abordar los conocimientos disciplinares que son contenidos conceptuales específicos de las demás unidades curriculares que constan en la estructura superficial del diseño. Por otro lado, teniendo en cuenta que esta unidad curricular sería dictada por profesores que no forman parte de la planta docente de los IFD, y que son capacitados especialmente por el Ministerio de Educación de la Provincia, es que podríamos inferir que la misma no cuenta con contenidos específicos explícitos porque éstos serían definidos directamente por la capacitación que el Ministerio otorgue a los docentes seleccionados para dictarla. 
De esta manera, puede pensarse que se le estaría quitando autonomía y poder de discusión y decisión a los IFD sobre esta unidad curricular.

A continuación analizaremos los espacios transversales que posee en calidad de innovaciones el nuevo DCJ: Itinerarios por el Mundo de la Cultura y Taller de Producción Pedagógica. Dichos espacios apuntan a la relación con el medio, ya que desde el Ministerio de Educación de la Provincia se postula que la formación no se produce dentro de un aula sino que se aprende en constante interacción con el medio socio-cultural. Esta postura se encuentra explicitada en la estructura profunda del diseño analizado.

Frente a los Itinerarios por el Mundo de la Cultura, los docentes de los IFD sostienen que, por un lado, resulta un buen aporte para la aproximación al medio socio-cultural por parte de los estudiantes. Por otro lado, consideran que dicho espacio no sería productivo para la formación docente si no es retrabajado dentro del aula. De este modo, el aprendizaje de los estudiantes de los IFD, a través de este espacio transversal, sería principalmente una experiencia personal y no suscitaría una reflexión colectiva.

El Taller de Producción Pedagógica se encontraría bajo las mismas circunstancias y críticas que el Itinerario por el Mundo de la Cultura ya que, si bien inserta al futuro docente a la realidad institucional desde el comienzo de su formación, éste no poseería las herramientas teóricas necesarias para enfrentar, analizar y generar alternativas ante una problemática educativa.

Otro de los espacios curriculares que generó opiniones encontradas entre los diferentes actores curriculares fue la modificación de los Trayectos de la Práctica con inserción en las instituciones educativas desde primer año. El Ministerio de Educación de la Provincia avala esta modificación ya que éste fue quien la diseñó. Los especialistas que formaban parte de la Comisión de Cambio Curricular apoyaban esta decisión argumentando que la práctica es la base de la formación. Sin embargo, los cientistas de la educación que también integraban dicha comisión no acordaban con esta forma de concebir la práctica debido a que no se trata sólo de zambullirse en ella sino que también es necesario re-trabajarla en el aula. Este último posicionamiento nos parece certero ya que la práctica docente no sería una práctica en sí misma, sino que requiere de reflexión a la luz de conceptos teóricos para modificar o transformar tanto la propia teoría como la práctica educativa.

En contraposición a esto, docentes y estudiantes de los IFD opinan favorablemente acerca de la implementación de dicho trayecto. En la mayoría de los casos, esto se justifica debido a la importancia que se le da a la inmersión en la práctica, pudiendo visualizar, de esta manera, la futura realidad laboral.

Finalmente, con respecto al DCJ analizado nos resulta pertinente desarrollar brevemente dos cuestiones relevantes a tener en cuenta a la hora de analizar todo proyecto curricular: si promueve un tratamiento disciplinar o interdisciplinar del conocimiento, y si es prescriptivo u orientador. 
En primer lugar, el Diseño Curricular para el Profesorado de Educación Primaria $N^{\circ} 528 / 09$ de la Provincia de Santa Fe no presenta espacios interdisciplinarios, los cuáles fueron reclamados por los IFD en el momento en que fueron consultados por la Comisión de Cambio Curricular y además, esta cuestión era una sugerencia que provenía desde el INFOD.

Si bien el diseño contiene espacios curriculares pensados disciplinarmente, el énfasis no estaría puesto en éstos sino en los espacios innovadores descriptos y explicados anteriormente. Éstos últimos son los que intentarían atribuirle al diseño flexibilidad y dinamismo, lo cuál conformaba uno de los objetivos que se habían planteado desde el Ministerio de Educación de la Provincia y era compartido por los especialistas que formaban parte de la Comisión de Cambio Curricular. No obstante, dicho objetivo no pudo plasmarse en el diseño curricular debido a los conflictos y tensiones que se desataron a lo largo del proceso de construcción entre los diferentes integrantes de dicha comisión.

Por todo lo analizado hasta aquí en relación a la estructura profunda del diseño y su correlato en la estructura superficial es que podríamos denominar al mismo como un diseño ecléctico debido a que finalmente es básicamente disciplinar y posee algunos espacios innovadores. Además, la estructura profunda del mismo sería coherente principalmente con estos últimos y no fundamentaría el abordaje disciplinar del resto del diseño.

La segunda característica importante para tratar en el análisis de todo proyecto curricular es si el mismo es prescriptivo u orientador. En el caso del diseño analizado, se explicita en su propia estructura superficial que es de carácter prescriptivo. No obstante, este diseño no prescribe criterios de evaluación ni define lo que entiende por la misma.

\section{Impactos e implicancias}

El presente eje pretende abrir puntas para futuras investigaciones a través del tratamiento de diversos impactos e implicancias que el nuevo Diseño Curricular para el Profesorado de Educación Primaria generó en la carrera docente, en los IFD y en sus actores institucionales en cuanto a su conocimiento e implementación.

Con respecto a los impactos que el diseño podría generar, uno de los miedos que circulaba en los IFD era que bajara la matrícula por la extensión de la carrera docente a cuatro años. Sin embargo, en el poco tiempo de implementación que posee este diseño parecería que la matrícula se mantuvo o aumentó mínimamente. Además, el $70 \%$ de los estudiantes encuestados de los IFD afirman que el nuevo DCJ mejora la calidad de la formación docente, jerarquizando, así, a la misma.

Otra cuestión que tuvo un fuerte impacto en los diferentes actores institucionales fue el desarrollo del nuevo diseño, lo cual generó tanto en los docentes como en los alumnos de los IFD, un estado de confusión y desorientación en 
relación a los espacios innovadores que el mismo plantea. Por un lado, dicho estado de confusión podría atribuirse a las modificaciones que todo cambio curricular conlleva. Por otro lado, podríamos dilucidar que esto se acrecentaría ante el hecho de que justamente los espacios innovadores que el diseño posee no estarían garantizados por el Ministerio de Educación de la Provincia y, por lo tanto, no se desarrollaron durante el primer año de implementación del mismo. De esta manera, dicha implementación parecería haber estado poco planificada, el currículum escrito no se habría podido concretar en el currículum real, en la cotidianeidad del aula.

Todo lo analizado y explicado hasta el momento nos abre múltiples interrogantes, desde cómo y quién/quiénes van a llevar adelante el adecuado desarrollo e implementación curricular del presente diseño, hasta de qué modo esto va a incidir en la formación de los futuros docentes santafesinos y en el desarrollo del Sistema Educativo Nacional en su totalidad.

\section{A modo de reflexión}

No es tarea fácil explicitar en esta breve reflexión final todo lo que aprendimos al realizar el Proyecto de Investigación que implicó un arduo trabajo tanto de investigación bibliográfica como en el campo y que dio origen al presente artículo.

Queremos manifestar lo fructífero que resultó el hecho trabajar en equipo ya que de esta forma se tejen discusiones y confrontaciones que nos llevan a enriquecer tanto nuestro conocimiento como nuestra subjetividad.

Desde el momento en que nos propusimos trabajar con la temática de la Formación Docente y, concretamente, con el controvertido proceso de Cambio Curricular para el Profesorado de Educación Primaria de la provincia de Santa Fe desarrollado durante el año 2008, supimos que no iba a ser tarea fácil. Sin embargo, a pesar de esto, llevamos adelante nuestro proyecto con entusiasmo y pasión, tratando de sortear de manera productiva cada obstáculo que se nos fue presentando en el camino.

Esperamos que el presente artículo aporte a clarificar lo sucedido en el proceso de cambio curricular analizado y que abra puntas para futuras investigaciones dentro del campo curricular. Campo en el que, una vez que nos introducimos, nos permite abrir otras miradas frente a la relación sociedadeducación así como también ante la realidad educativa.

\section{Notas Bibliográficas}

(1) Brovelli, M. (2008) "Los cambios curriculares: ¿Una posibilidad de mejora en la formación docente y de encuentro de los actores responsables o del mantenimiento del status quo y del desencuentro?", en Revista de la Escuela de Ciencias de la Educación Año 4 N ${ }^{\circ}$ 3; Laborde Editor; Rosario. 


\section{Referencias Bibliográficas}

- Brovelli, M. (2008) “Los cambios curriculares: ¿Una posibilidad de mejora en la formación docente y de encuentro de los actores responsables o del mantenimiento del status quo y del desencuentro?", en Revista de la escuela de Ciencias de la Educación Año 4 № 3; Laborde Editor; Rosario.

- Brovelli, M. (2006) Cambio e innovaciones educativas: representaciones y contextos, en Revista de la escuela de Ciencias de la Educación Año 2 - N 1; Laborde Editor; Rosario.

- Brovelli, M. "Políticas de cambio curricular. Ámbitos de decisión y concreción curricular"; Ficha de cátedra.

- Contreras, J. (1990) Enseñanza, currículum y profesorado; Akal Universitaria; Madrid;

- $\quad$ Cullen, C. (1993) Interdisciplinariedad en Ciencias Sociales; Paidós.

- $\quad$ De Alba, A. (1988) En torno a la noción de currículum.

- $\quad$ Frigerio, G; Poggi, M y Tiramonti, G; (1992) "Actores, instituciones y conflictos", en Las instituciones educativas. Cara y ceca. Elementos para su comprensión; Troquel educación; Serie FLACSO-Acción; Buenos Aires.

- Gimeno Sacristán, J; (1977) "Reformas educativas: utopía, retórica y práctica”, "El perfeccionamiento como desarrollo de la profesionalidad docente", en Docencia y Cultura Escolar. Reformas y modelo educativo; Lugar Editorial; Buenos Aires.

- $\quad$ Gimeno Sacristán, J.; (1986) "El profesor y la formación del profesorado", en La enseñanza: su teoría y su práctica; Ed. Akal; Madrid.

- $\quad$ Giroux, H; (1990) Los profesores como intelectuales; Paidós; Buenos Aires.

- Kemmis, S.; (1988) El currículo: más allá de la Teoría de la Reproducción; Morata; Madrid.

- $\quad$ Obiols, G. y Obiols, S. (1992) "Modernidad y posmodernidad: elementos para entender un debate"; en Adolescencia y posmodernidad en la escuela secundaria.

- Pérez Gómez, A. (1992) “La función y la formación del profesor/a en la enseñanza para la comprensión. Diferentes perspectivas", en Comprender y transformar la enseñanza; Morata; Madrid.

- Schon, D. (1992) La formación de Profesionales Reflexivos; Paidós; Barcelona.

\section{Documentos}

- Plan Nacional de Formación Docente

- Ley Nacional de Educación № 26.206/06

- Lineamientos curriculares nacionales para la formación docente inicial

- Diseño Curricular de Formación Docente para el Nivel Primario en la Provincia de Santa Fe (http://www.portal.santafe.gov.ar/index.php/web/content/view/full) 